\title{
Using turn taking to achieve intertemporal cooperation and symmetry in infinitely repeated $2 \times 2$ games
}

\author{
Sau-Him Paul Lau • Vai-Lam Mui
}

Published online: 10 April 2011

(C) The Author(s) 2011. This article is published with open access at Springerlink.com

\begin{abstract}
Turn taking is observed in many field and laboratory settings captured by various widely studied $2 \times 2$ games. This article develops a repeated game model that allows us to systematically investigate turn-taking behavior in many $2 \times 2$ games, including the battle of the sexes, the game of chicken, the game of common-poolresources assignment, and a particular version of the prisoners' dilemma. We consider the "turn taking with independent randomizations" (TTIR) strategy that achieves three objectives: (a) helping the players reach the turn-taking path, (b) resolving the question of who takes the good turn first, and (c) deterring defection. We determine conditions under which there exists a unique TTIR strategy profile that can be supported as a subgame-perfect equilibrium. We also show that there exist conditions under which an increase in the "degree of conflict" of the stage game leads to a decrease in the expected number of periods in reaching the turn-taking path.
\end{abstract}

Keywords Conflict - Coordination · Turn taking - Intertemporal cooperation

JEL Classification $\quad \mathrm{C} 70 \cdot \mathrm{C} 72$

\section{Introduction}

Turn-taking behavior is observed in a variety of field and laboratory settings. An example of turn taking concerns the use of common-pool resources (CPRs) such as fisheries,

S.-H. P. Lau (凷

School of Economics and Finance, University of Hong Kong, Pokfulam, Hong Kong e-mail: laushp@hku.hk

Department of Economics, Monash University, Clayton, VIC 3800, Australia e-mail: Vai-Lam.Mui@BusEco.Monash.edu.au 
irrigation systems, and forests. In communities that depend heavily on such resources for their economic livelihood, failure to resolve problems related to the use of these resources can lead to significant welfare loss and violent conflicts. One illustration of the conflicts studied in the CPR literature is the game of CPR assignment in Ostrom et al. (1994, pp. 58-61). In this game, two fishermen independently decide to go to one of two fishing spots in their community. The good spot has a value of $h$, and the bad spot has a value of $l$, where $h>l>0$. If the two fishermen choose different spots, each will obtain the respective value of the spot. If they choose the same spot, they will split the value of the spot equally.

Both coordination and conflict elements (Friedman 1994, pp.7-8) are present in this game. To maximize total surplus, the fishermen should choose different fishing spots; however, they may end up at the same spot if there is no coordination. Moreover, a conflict element is present because while both players prefer going to different spots rather than to the same spot, each prefers to go to the good spot, while the other choosing the bad one. If this game is played repeatedly, one might expect that some sort of rotation scheme, in which the fishermen take turns going to the good spot, will eventually be adopted. In fact, Berkes (1992) reports that fishermen in Turkey employ a turn-taking scheme to allocate fishing spots. Rotation schemes in the use of irrigation system have been observed in Spain and the Philippines (Ostrom 1990).

Turn taking is also observed in other settings. For example, faculty members in a department may use turn taking to resolve the question of who will serve as the departmental representative on a university committee. Soldiers in a military operation often take turns to serve as the "point man" in dangerous missions (Bergerud 1993). These examples possess the feature of the repeated best-shot public good game, in which the socially available amount of public good is the maximum of individual contributions (Harrison and Hirshleifer 1989). Turn taking in another game-the repeated battle of the sexes-has also been mentioned in the literature, dating back to Luce and Raiffa (1957).

Researchers have also observed turn-taking behavior in laboratory games, ranging from a repeated CPR appropriation externality game (Prisbrey 1992, Chap. 1), to the repeated best-shot public good game (Bornstein et al. 1997) and the repeated traffic route choice game (Helbing et al. 2005). ${ }^{1}$ In all three studies, eventual turn taking with initial coordination failure is widely observed. ${ }^{2}$ Furthermore, all three studies suggest that when the stage game is a symmetric $2 \times 2$ game (i.e., with two players and two actions), turn taking often takes the form of single-period alternation between the players.

The literature reviewed above suggests that turn taking behavior is important in many settings captured by widely studied games with binary choices, and the

\footnotetext{
${ }^{1}$ Bornstein et al. (1997) call the game used in their experiments a game of chicken, but they mention that the payoff structure is qualitatively similar to but slightly different from the traditional game of chicken. As seen from Harrison and Hirshleifer (1989), it is more appropriate to call the game studied in Bornstein et al. (1997) a best-shot public good game.

2 All these laboratory studies concern games with complete information, which is the case considered in this article. Kaplan and Ruffle (2007) study a repeated entry game with incomplete information, in which a player's payoff of entry depends on private information. They find that many subjects take turns to enter to avoid efficiency loss due to simultaneous entry.
} 
Table 1 Strategies and payoffs of the stage game

(a) The $h$ and $l$ specification

\begin{tabular}{lll}
\hline $1 \backslash 2$ & Tough & Soft \\
\hline $\begin{array}{l}\text { Tough } \\
\text { Soft }\end{array}$ & $(t, t)$ & $(h, l)$ \\
(b) The $\lambda$ and $\theta$ specification & $(l, h)$ & $(s, s)$ \\
\hline $1 \backslash 2$ & & \\
\hline Tough & Tough & Soft \\
Soft & $(t, t)$ & $\left(\frac{\theta \lambda}{1+\theta}, \frac{\lambda}{1+\theta}\right)$ \\
\hline
\end{tabular}

experimental evidence suggests that delay in reaching successful turn taking is common. This raises the question of how differences in the structure of the game can affect turn-taking behavior, including the length of delay in reaching turn taking. This article analyzes turn taking in a repeated symmetric $2 \times 2$ game that nests many well-known games as special examples. In the stage game of the repeated game analyzed in this article, each player chooses between Tough $(T)$ and Soft $(S)$. As seen in the top panel of Table 1, each player obtains payoff $t$ if both choose the same action $T$, and each obtains payoff $s$ if both choose $S$. A player obtains payoff $h$ if she chooses $T$ and the other player chooses $S .{ }^{3}$ In this case, the other player obtains a lower payoff $l$. We assume that $h+l>\max \{2 s, 2 t\}$, so that the two asymmetric outcomes $(T, S)$ and $(S, T)$ maximize total payoffs in this game.

To capture the kind of "trial and error" process that players use to resolve the coordination and conflict problems, as well as the observation that turn taking often takes the form of single-period alternation, we consider a simple strategy-to be called the "turn taking with independent randomizations" (TTIR) strategy - in this paper. Players using this strategy randomize between $T$ and $S$ in the beginning period and also if either of the symmetric outcomes is observed in the preceding period, or rotate between the two asymmetric outcomes if one of these outcomes is reached in the preceding period.

We determine the conditions under which a TTIR strategy profile can be supported as a subgame-perfect equilibrium. We find that whenever there exists a TTIR strategy profile that constitutes an equilibrium, it must be unique and symmetric (i.e., each player adopts the same randomization probability in equilibrium). Moreover, there are systematic differences between the games in which the two asymmetric outcomes are Nash equilibria in the stage game (to be called the accommodating case), and those with $(T, T)$ as the unique Nash equilibrium of the stage game (to be called the mutualtough case). We find that the TTIR strategy can be supported as an equilibrium for any

\footnotetext{
3 We use female pronouns throughout this article; their male counterparts get their turn in a companion paper.
} 
discount factor in the accommodating case, but it can be supported as an equilibrium in the mutual-tough case if and only if the players are sufficiently patient.

In addition, we derive two sets of testable implications. First, we study the equilibrium delay. Stimulated by the results in Lau and Mui (2008) regarding the repeated battle of the sexes, we define the degree of conflict $(\theta)$ of the stage game as the ratio of the two players' payoffs at an asymmetric outcome $\left(\theta=\frac{h}{l}\right)$. It is natural to conjecture that equilibrium delay is increasing in the degree of conflict, since a higher degree of distributional conflict induces the players to behave more aggressively by choosing Tough with a higher probability in the initial periods. We find that this conjecture is correct for the mutual-tough case. However, for the accommodating case, there are games (such as the game of chicken) with the counter-intuitive feature that equilibrium delay is not always increasing in the degree of conflict. Second, for the mutual-tough case, we examine how changes in the primitives of the stage game affect the difficulty in supporting the TTIR strategy as an equilibrium through their effects on the critical discount factor. Overall, the results suggest that both the difficulty in sustaining turn-taking behavior, and how the expected length of delay is affected by the primitives of the game, depend crucially on whether the two asymmetric outcomes are Nash equilibria of the stage game.

We are not aware of any prior contribution that develops a unified analysis of turntaking behavior in widely studied $2 \times 2$ games. There are, however, other work that feature turn-taking strategies. Our article is related to several papers analyzing various $2 \times 2$ games. ${ }^{4}$ Motivated by power sharing arrangements in politics, Ward (1998) studies conditions under which players will not defect from turn taking in the repeated game of chicken and a version of the repeated prisoners' dilemma. Ward (1998), however, does not provide an equilibrium analysis of how players resolve the questions of how to get onto a turn-taking path and who has the good turn first. This article addresses these issues.

Lau and Mui (2008) consider turn taking in the repeated battles of the sexes, and show how a comparison of the "equilibrium" degree of conflict in the repeated battles of the sexes with its (exogenous) degree of conflict in the stage game provides an intuitive way of understanding how turn taking facilitates intertemporal cooperation. This article builds on some idea of that paper, but there are also substantial differences. This article analyzes many well-known games, and establish the key differences between the accommodating and mutual-tough cases, whereas Lau and Mui (2008) only study the repeated battles of the sexes which is an example of the accommodating case. This article allows for the possibility that players may randomize with different probabilities, and show that whenever there exists a TTIR profile that constitutes an equilibrium, it is unique and symmetric. This is more general than the result in Lau and Mui (2008) that whenever there exists an equilibrium symmetric TTIR strategy profile, it is unique. Moreover, this article performs comparative statics analysis that is not the focus of Lau and Mui (2008).

\footnotetext{
4 Outside the context of $2 \times 2$ games, researchers have studied collusive strategies that have turn-taking features in repeated auctions and repeated Bertrand games. See, for example, Skrzypacz and Hopenhayn (2004) and Athey and Bagwell (2008) and the references cited there.
} 
Our article is complementary to the work by Bhaskar (2000), who considers the egalitarian convention (which equalizes, as far as possible, the realized payoffs of the two players) in the repeated battle of the sexes and the repeated game of chicken. ${ }^{5}$ He finds that the efficient symmetric equilibrium can be supported by the equalitarian convention, which involves the use of time-varying strategies and has turn-taking features. Bhaskar's result is an important normative finding. However, experimental studies suggest that turn taking in symmetric $2 \times 2$ games often takes the form of one-period alternation instead of time-varying sequences (Prisbrey 1992; Bornstein et al. 1997; Helbing et al. 2005). Another implication of the strategies used in Bhaskar (2000) is that changes in the parameter values of the game do not affect the expected length of delay. The positive approach of our work is complementary to the normative approach of Bhaskar (2000), in that the TTIR strategy considered in this article assumes that turn taking takes the form of one-period alternation, and we aim to derive predictions regarding how changes in the parameter values of the game affect the expected length of delay.

This article is organized as follows. Section 2 introduces the model. Section 3 describes the TTIR strategy and the associated incentive conditions. Section 4 studies how the TTIR strategy can be supported as a subgame-perfect equilibrium for two major cases of the game. Section 5 derives comparative static results, and Sect. 6 concludes. Proof of Proposition 1 is reported in the Appendix, and all other proofs are available upon request.

\section{The model}

We consider a symmetric infinite-horizon repeated game with discounting. In every period, each of the two players (called 1 and 2) chooses (perhaps randomly) between two actions: playing $T$ or playing $S$. When making a decision in period $n$, player $i$ maximizes her intertemporal payoff (which is the discounted sum of the stream of her current and future single-period payoffs):

$$
\sum_{m=n}^{\infty} \delta^{m-n} U_{i}\left(x_{1 m}, x_{2 m}\right)
$$

\footnotetext{
5 Bhaskar (2000) considers the following strategy. Suppose that the realized outcome is $(T, S)$ when an asymmetric outcome occurs for the first time, with player 1's current-period payoff of $h$ exceeding player 2 's payoff of $l$. To implement the egalitarian convention, the players choose $(S, T)$ in succeeding periods until the intertemporal payoff of player 2 exceeds that of player 1 . At this point, the players switch to playing Footnote 5 continued

$(T, S)$ until player 1's intertemporal payoff exceeds player 2's, and so on. This strategy is more complicated than the TTIR strategy with one-period alternation. Such time-varying sequences induce a different continuation value than one-period alternation, and hence lead to a different probability of randomization in the beginning of the game. Computational results suggest that the use of time-varying sequences leads to a modest efficiency gain compared to one-period alternation. The relatively small gain, and the complexity involved, may explain why such time-varying pattern of turn taking is not much observed in laboratory studies.
} 
where $\delta \in(0,1)$ is the common discount factor, $x_{i m}\left(x_{i m}=T\right.$ or $\left.S\right)$ is the choice of player $i$ at period $m$, and $U_{i}\left(x_{1 m}, x_{2 m}\right)$ is the current-period payoff of player $i$ when player 1 chooses $x_{1 m}$ and player 2 chooses $x_{2 m}$. The players' payoffs in the stage game are represented by

$$
\begin{array}{cc}
U_{i}(T, T)=t, & U_{i}(S, S)=s, \\
U_{1}(T, S)=U_{2}(S, T)=h, & U_{1}(S, T)=U_{2}(T, S)=l,
\end{array}
$$

where $i=1,2$, and $h, l, s$, and $t$ are finite real numbers. (Whenever there is no confusion, the time subscript is ignored.) Payoffs are common knowledge. Each player observes both players' actions in earlier periods, but not how the other player randomizes.

In this article, we assume

$$
\begin{aligned}
h & >l, \\
h+l & >2 s,
\end{aligned}
$$

and

$$
h+l>2 t \text {. }
$$

Since interesting turn-taking behavior consists of good and bad turns, we assume (3) which allows for asymmetric outcomes in the stage game. ${ }^{6}$ If the two players choose different actions, their payoffs will be different under assumption (3). Without loss of generality, we assume that $h$ ("high") is larger than $l$ ("low"). The specification in (3) implicitly defines the labels $T$ and $S$ for any given game. For example, $T$ represents Good Spot and $S$ represents Bad Spot for the game of CPR assignment. On the other hand, assumptions (4) and (5) ensure that the sum of the players' payoffs in the two asymmetric outcomes is higher than that in the two symmetric outcomes.

Summing up, our focus is the class of infinitely repeated games with (1) to (5), and we shall hereafter denote a game in this class as $G_{\infty}$. The analysis turns out to differ for two mutually exclusive possibilities (the accommodating case $t<l$, or the mutualtough case $t>l$ ), and the differences can be traced to the different pure-strategy equilibrium (or equilibria) in the stage game. Before analyzing these two cases, it is helpful to summarize well-known results about the corresponding one-shot games.

When $t<l$, the two asymmetric joint-payoff-maximizing outcomes $(T, S)$ and $(S, T)$ are pure-strategy equilibria of the stage game. (There is also a symmetric mixed-strategy equilibrium.) Key examples for this case include (a) battle of the sexes (with $h>l>t=s$ ), (b) the version of game of chicken $(h>s>l>t)$ with $h+l>2 s$, (c) the best-shot public good game (with $h>s=l>t$ ), and (d) a particular version of the game of CPR assignment (Ostrom et al. 1994) when $h<2 l$, that is, when the bad spot is not too inferior compared to the good spot.

\footnotetext{
6 Assumption (3) eliminates the pure coordination game from our analysis, but many widely studied games are consistent with this assumption.
} 
On the other hand, when $t>l$, each player's dominant strategy in the stage game is to choose Tough, and the two asymmetric outcomes are not Nash equilibria. Examples for this case include (a) a particular version of the prisoners' dilemma (see, for example, Dixit and Skeath 1999, Fig. 11.2), ${ }^{7}$ (b) another version of the game of CPR assignment when $h>2 l$, that is, when the good spot is "sufficiently more attractive" than the bad spot, and (c) the game of CPR appropriation externality (with $h>t>s=l$ and $h+l>2 t$ ) studied by Prisbrey (1992) and Ostrom et al. (1994, Table 3.4c).

\section{TTIR strategy}

In this article, we study turn taking when there is no communication between the players. Turn taking is observed in experimental and field settings in which communication is not possible, but it is also observed in environments in which the players are able to communicate. We think it is useful to first study turn taking in an environment in which the players cannot communicate. In this environment, any benefit accrued to the players in mitigating conflict and enhancing coordination is purely due to turn taking and not to communication. In this setting, two related coordination and conflict problems have to be resolved. First, how do the players get onto either one of the two turn-taking sequences $\{\ldots,(T, S),(S, T),(T, S),(S, T), \ldots\}$ and $\{\ldots,(S, T),(T, S),(S, T),(T, S), \ldots\}$ ? Second, who gets to start with the good turn first?

The experimental evidence reviewed above suggest that in the absence of preplay communication, players go through some kind of trial and error process to resolve the coordination and conflict problems. To capture this process in a parsimonious way, we consider the TTIR strategy that specifies the following: (a) In the beginning period, the players will independently randomize between $T$ and $S$. Denote player $i$ 's probability of choosing $T$ as $p_{i}(i=1,2)$. For meaningful TTIR strategy, $p_{1}$ and $p_{2}$ are restricted to lie in the open interval $(0,1)$. (b) As long as the randomization yields the symmetric outcome of either $(T, T)$ or $(S, S)$, the randomization phase will continue. (c) Whenever randomization succeeds in getting the players to the asymmetric outcome of either $(T, S)$ or $(S, T)$, the game will switch to the turn-taking phase in which each player chooses the action her opponent took in the previous period. If no player defects from this strategy, the turn-taking phase will continue. (d) Any defection by a player (or by both players) during the turn-taking phase will trigger a switch back to the randomization phase, and this randomization phase will continue until randomization succeeds in getting the players to the asymmetric outcome of either $(T, S)$ or $(S, T)$

\footnotetext{
7 Dixit and Skeath (1999, Figs 11.1, 11.2) consider two games to study the issue of collective action in building an irrigation project. Each of these games satisfies the two usual key properties of the prisoners' dilemma (with $h>s>t>l$ ), namely, that Defect is the dominant strategy for both players, and the outcome (Cooperate, Cooperate) Pareto dominates (Defect, Defect). In the first game (with $h+l<2 s$ ), (Cooperate, Cooperate) is the joint-payoff-maximizing outcome. This is the game that most analyses of the prisoners' dilemma focus on, and we refer to it as the "standard" prisoners' dilemma. In the other game Footnote 7 continued

(their Fig. 11.2, with $h+l>2 s$ ), the two asymmetric outcomes - that is, when one player defects and the other cooperates-maximize the players' total payoff. This non-standard prisoners' dilemma has also been studied by Ward (1998).
} 
again. ${ }^{8}$ (e) Once randomization succeeds in getting the players to either asymmetric outcome, the players will again behave according to steps (c) and (d).

We determine the conditions under which the TTIR strategy can be supported as a subgame-perfect equilibrium. Whether the TTIR strategy profile constitutes an equilibrium depends on the incentive conditions in the randomization and turn-taking phases, which we now consider.

\subsection{Players' behavior in the turn-taking phase}

Define $V^{\mathrm{H}}$ (resp. $V^{\mathrm{L}}$ ) as a player's intertemporal payoff at a period in which she plays Tough (resp. Soft) and her opponent plays Soft (resp. Tough), with the expectation that both players will choose the equilibrium TTIR strategy in the future. Define $V_{i}^{*}(i=1,2)$ as player $i$ 's intertemporal payoff in the randomization phase (including the beginning period), with the expectation that both players will choose the equilibrium TTIR strategy in the future. ${ }^{9}$

The two value functions of the turn-taking phase are given by

$$
V^{\mathrm{H}}=h+\delta V^{\mathrm{L}}=\frac{h+\delta l}{1-\delta^{2}},
$$

and

$$
V^{\mathrm{L}}=l+\delta V^{\mathrm{H}}=\frac{l+\delta h}{1-\delta^{2}} .
$$

To ensure that (6) and (7) are well defined, we need to check two no-deviation conditions for each player in the turn-taking phase, one at a player's good turn and the other at her bad turn.

If players $i$ and $j(i, j=1,2$ and $i \neq j)$ in the previous period chose $S$ and $T$, respectively, then, player $i$ will not deviate in the current period when

$$
V^{\mathrm{H}}-\left(s+\delta V_{i}^{*}\right)=(h-s)+\delta\left(V^{\mathrm{L}}-V_{i}^{*}\right)>0 .
$$

Similarly, if the actions of players $i$ and $j$ in the previous period were $T$ and $S$, respectively, then player $i$ will not deviate in the current period when

$$
V^{\mathrm{L}}-\left(t+\delta V_{i}^{*}\right)=(l-t)+\delta\left(V^{\mathrm{H}}-V_{i}^{*}\right)>0 .
$$

\footnotetext{
8 Our analysis focuses on the specification in which defection during the turn-taking phase will trigger a switch back to the randomization phase. As explained in Sect. 4.3, however, our results are robust to other punishment strategies.

9 Note that this notation implicitly allows for the possibility that the two players' intertemporal payoffs in the randomization phase are different. This can occur if each player uses a different randomization probability in equilibrium.
} 
Table 2 Strategies and intertemporal payoffs at the beginning of the repeated game

\begin{tabular}{lll}
\hline $1 \backslash 2$ & Playing tough at period 0 & Playing soft at period 0 \\
\hline Playing tough at period 0 & $\left(t+\delta V_{1}^{*}, t+\delta V_{2}^{*}\right)$ & $\left(V^{\mathrm{H}}, V^{\mathrm{L}}\right)$ \\
Playing soft at period 0 & $\left(V^{\mathrm{L}}, V^{\mathrm{H}}\right)$ & $\left(s+\delta V_{1}^{*}, s+\delta V_{2}^{*}\right)$ \\
\hline
\end{tabular}

\subsection{Players' behavior in the randomization phase}

Next, we examine the randomization phase (including the beginning period). If both players use the TTIR strategy, it is easy to see that the game will remain in the randomization phase in the next period if and only if both players choose the same action in the current period.

For subsequent analysis, it is helpful to define

$$
V_{i}\left(p_{i}, p_{j}\right)=\frac{p_{i} p_{j} t+\left(1-p_{i}\right)\left(1-p_{j}\right) s+p_{i}\left(1-p_{j}\right) V^{\mathrm{H}}+\left(1-p_{i}\right) p_{j} V^{\mathrm{L}}}{1-\delta\left[p_{i} p_{j}+\left(1-p_{i}\right)\left(1-p_{j}\right)\right]},
$$

which gives player $i$ 's intertemporal payoff at the randomization phase when players $i$ and $j$ choose Tough with probability $p_{i}$ and $p_{j}$, respectively, since this phase will continue with probability $p_{i} p_{j}+\left(1-p_{i}\right)\left(1-p_{j}\right)$ in the next period.

Denote player $i$ 's equilibrium probability of choosing $T$ in the randomization phase as $p_{i}^{*}$. In the randomization phase, player $i$ chooses $p_{i}^{*}$ to ensure that the other player is indifferent between playing $T$ and $S$. It can be deduced from Table 2 that the players' equilibrium randomization probabilities $\left(p_{1}^{*}\right.$ and $p_{2}^{*}$ ) and equilibrium intertemporal payoffs $\left(V_{1}^{*}\right.$ and $\left.V_{2}^{*}\right)$ are jointly determined by

$$
V_{i}^{*}=p_{j}^{*}\left(t+\delta V_{i}^{*}\right)+\left(1-p_{j}^{*}\right) V^{\mathrm{H}}=p_{j}^{*} V^{\mathrm{L}}+\left(1-p_{j}^{*}\right)\left(s+\delta V_{i}^{*}\right),
$$

where $i, j=1,2$ and $i \neq j$. Note that $V_{i}^{*}$ in (11) is related to the function $V_{i}\left(p_{i}, p_{j}\right)$ in (10) according to

$$
V_{i}^{*}=V_{i}\left(p_{i}^{*}, p_{j}^{*}\right)
$$

To examine the conditions characterizing the equilibrium randomization probabilities, we combine (10), (10a), and the second equality of (11) to obtain

$$
p_{j}^{*}=\frac{V^{\mathrm{H}}-s-\delta V_{i}^{*}}{\left(V^{\mathrm{H}}-s-\delta V_{i}^{*}\right)+\left(V^{\mathrm{L}}-t-\delta V_{i}^{*}\right)}
$$




$$
=\frac{V^{\mathrm{H}}-s-\delta\left\{\frac{p_{i}^{*} p_{j}^{*} t+\left(1-p_{i}^{*}\right)\left(1-p_{j}^{*}\right) s+p_{i}^{*}\left(1-p_{j}^{*}\right) V^{\mathrm{H}}+\left(1-p_{i}^{*}\right) p_{j}^{*} V^{\mathrm{L}}}{1-\delta\left[p_{i}^{*} p_{j}^{*}+\left(1-p_{i}^{*}\right)\left(1-p_{j}^{*}\right)\right]}\right\}}{V^{\mathrm{H}}+V^{\mathrm{L}}-s-t-2 \delta\left\{\frac{p_{i}^{*} p_{j}^{*} t+\left(1-p_{i}^{*}\right)\left(1-p_{j}^{*}\right) s+p_{i}^{*}\left(1-p_{j}^{*}\right) V^{\mathrm{H}}+\left(1-p_{i}^{*}\right) p_{j}^{*} V^{\mathrm{L}}}{1-\delta\left[p_{i}^{*} p_{j}^{*}+\left(1-p_{i}^{*}\right)\left(1-p_{j}^{*}\right)\right]}\right\}},
$$

where $i, j=1,2$ and $i \neq j$, and $V^{\mathrm{H}}$ and $V^{\mathrm{L}}$ depend on parameters $\delta, h$, and $l$ according to (6) and (7). That is, $p_{1}^{*} \in(0,1)$ and $p_{2}^{*} \in(0,1)$ are the equilibrium randomization probabilities if they satisfy simultaneously the two equations defined by (12).

Condition (12) can be interpreted as follows. If the no-deviation conditions (8) and (9) are satisfied, then player $j$ 's equilibrium randomization probability $\left(p_{j}^{*}\right)$ in the current period is given by the middle term of (12), which involves $V_{i}^{*}$, since the game may remain in the randomization phase in the next period. As given by (10a), player $i$ 's continuation payoff $V_{i}^{*}$ depends on equilibrium randomization probabilities $p_{i}^{*}$ and $p_{j}^{*}$ (in the future). Thus, one can think of $p_{j}^{*}(j=1,2)$ on the left-hand side of (12) as the probability that player $j$ choose $T$ in the current period during the randomization phase, and $p_{1}^{*}$ and $p_{2}^{*}$ on the right-hand side as the probabilities that the two players choose $T$ in the future (if the game remains in the randomization phase). The two equilibrium conditions in (12) are consistency conditions between current and future randomization probabilities of this infinitely repeated game.

\section{Supporting the TTIR strategy as an equilibrium}

The TTIR strategy can be supported as a subgame-perfect equilibrium of the repeated game $G_{\infty}$ if there exists $p_{1}^{*} \in(0,1)$ and $p_{2}^{*} \in(0,1)$ that simultaneously satisfy the no-deviation conditions (8) and (9) during the turn-taking phase and the equilibrium randomization conditions (12) during the randomization phase. The equilibrium profile is unique if there exists only one pair of $p_{1}^{*} \in(0,1)$ and $p_{2}^{*} \in(0,1)$ that satisfies these conditions. It is symmetric if $p_{1}^{*}=p_{2}^{*}$.

Our formulation allows for asymmetric TTIR strategies. In the Appendix, we show that no asymmetric TTIR profile can be supported as an equilibrium in the symmetric game $G_{\infty} \cdot{ }^{10}$ To simplify exposition, we focus on symmetric TTIR strategies (with $p_{1}=p_{2}$ ) in the main text, and report the discussion of asymmetric TTIR strategies in the Appendix.

It is natural to expect that the no-deviation condition (8) always holds, since a player has no incentive to defect when she is supposed to take her good turn. The following Lemma is useful in establishing condition (8).

Lemma 1 For the repeated game $G_{\infty}$,

$$
V^{\mathrm{H}}-\left[s+\delta V_{i}(p, p)\right]>0
$$

\footnotetext{
10 In particular, we show that no pair of asymmetric randomization probabilities $\left(p_{1}, p_{2}\right)$ with $p_{1} \neq p_{2}$ can satisfy the two conditions in (12), even if they satisfy conditions (8) and (9).
} 
for all $\delta \in(0,1)$ and all $p \in(0,1)$.

Lemma 1 implies that if there exists $p^{*}$ such that $p_{1}^{*}=p_{2}^{*}=p^{*}$ constitutes an equilibrium, then the no-deviation condition (8) is always non-binding.

Our remaining tasks are to examine under what circumstances the no-deviation condition (9) at a player's bad turn holds, and to study the equilibrium randomization conditions in (12). The analysis differs for the accommodating and mutual-tough cases, as the underlying structure of the game is different in these two cases.

\subsection{The accommodating case $(t<l)$}

For the accommodating case, we obtain the following Lemma regarding the no-deviation condition (9) when a player is supposed to take her bad turn.

Lemma 2 For the repeated game $G_{\infty}$ with $t<l$,

$$
V^{\mathrm{L}}-\left[t+\delta V_{i}(p, p)\right]=(l-t)+\delta\left[V^{\mathrm{H}}-V_{i}(p, p)\right]>0
$$

for all $\delta \in(0,1)$ and all $p \in(0,1)$.

Lemma 2 implies that for the accommodating case, if there exists a symmetric TTIR strategy profile that constitutes an equilibrium, then the no-deviation condition (9) is always non-binding. A player will not defect when she is supposed to take her bad turn, because by adhering to the equilibrium strategy, she will have both a current gain of $l-t$ and a future gain of $\delta\left(V^{\mathrm{H}}-V_{i}^{*}\right)$.

With Lemma 1 and Lemma 2, we show in Proposition 1 that in the accommodating case of game $G_{\infty}$, for all $\delta \in(0,1)$, there exists a unique and symmetric TTIR profile that can be supported as an equilibrium.

Proposition 1 For the repeated game $G_{\infty}$ with $t<l$ and $\delta \in(0,1)$, there exists a unique TTIR strategy profile that can be supported as a subgame-perfect equilibrium. The TTIR strategy profile is symmetric, with the equilibrium randomization probability of each player given by

$$
p^{*}=\frac{-c}{b}
$$

if $a=0$, or by

$$
p^{*}=\frac{-b-\sqrt{b^{2}-4 a c}}{2 a}
$$

if $a \neq 0$, where coefficients $a, b$ and $c$ are related to the discount factor and stage-game payoff parameters according to

$$
\begin{aligned}
& a=\delta[(1+\delta)(t-s)-(h-l)], \\
& b=\left(1-\delta^{2}\right) t+(1+\delta)^{2} s-h-(1+2 \delta) l,
\end{aligned}
$$


and

$$
c=h+\delta l-(1+\delta) s .
$$

\subsection{The mutual-tough case $(t>l)$}

As the proof for the accommodating case is relatively straightforward, it may appear that it would also be easy to obtain the conditions under which (9) holds for the mutual-tough case by expressing $V^{\mathrm{L}}$ and $V_{i}^{*}$ in (9) in terms of $\delta, h, l, s$, and $t$. For example, one may think of using the closed-form solution for $p^{*}$ similar to (13) and (14) for the accommodating case. However, while (13) and (14) hold for all discount factors $\delta \in(0,1)$ for the accommodating case, they will only hold for sufficiently high discount factors for the mutual-tough case, as we shall show later. Therefore, we need to first determine the range of discount factors in which the TTIR strategy can be supported as an equilibrium and (13) and (14) hold for the mutual-tough case.

In the analysis of the mutual-tough case, we proceed as follows. We first conjecture that there exists a critical discount factor $\delta_{\mathrm{TT}} \in(0,1)$ such that for $\delta \in\left(\delta_{\mathrm{TT}}, 1\right)$, there exists a unique symmetric TTIR strategy profile that can be supported as an equilibrium. We then show that for all $\delta \in\left(\delta_{\mathrm{TT}}, 1\right)$, if there exists a unique symmetric TTIR strategy profile that constitutes an equilibrium, the function $p^{*}(\delta)$ must be strictly decreasing in $\delta$. These results enable us to determine the value of $\delta_{\mathrm{TT}}$ as a function of the payoff parameters of the stage game. We then close our proof by showing that for all $\delta \in\left(\delta_{\mathrm{TT}}, 1\right)$, in fact there exists a unique TTIR strategy profile that constitutes an equilibrium and it is symmetric.

Assuming first that for all $\delta \in\left(\delta_{\mathrm{TT}}, 1\right)$, there exists a unique symmetric TTIR strategy profile that can be supported as an equilibrium (with $p_{i}^{*}=p_{j}^{*}=p^{*}$ and $V_{i}^{*}=V_{j}^{*}=V^{*}$ ), we investigate how the equilibrium randomization probability changes when only the discount factor changes. The partial derivative $\frac{\partial p^{*}}{\partial \delta}$ is given in (19) below. Manipulating various terms in (19) leads to the following Lemma regarding the monotonicity of $p^{*}$ with respect to $\delta .{ }^{11}$

Lemma 3 For the repeated game $G_{\infty}$ with $t>l$, if for all $\delta \in\left(\delta_{\mathrm{TT}}, 1\right)$, there exists a unique symmetric TTIR strategy profile that can be supported as a subgame-perfect equilibrium, then $p^{*}$ satisfies

$$
0.5<p^{*}<1
$$

and

$$
\frac{\partial p^{*}}{\partial \delta}=\frac{\left(1-p^{*}\right)\left[\frac{\partial V^{\mathrm{H}}}{\partial \delta}-\delta \frac{\partial V^{*}}{\partial \delta}-V^{*}\right]-p^{*}\left[\frac{\partial V^{\mathrm{L}}}{\partial \delta}-\delta \frac{\partial V^{*}}{\partial \delta}-V^{*}\right]}{\left(V^{\mathrm{H}}-s-\delta V^{*}\right)+\left(V^{\mathrm{L}}-t-\delta V^{*}\right)+\delta\left(1-2 p^{*}\right) \frac{\partial V^{*}}{\partial p^{*}}}
$$

\footnotetext{
11 On the other hand, for the accommodating case, we have found examples in which the monotonicity property of $p^{*}$ with respect to $\delta$ does not hold.
} 
is negative.

Lemma 3 is a crucial step in obtaining the critical discount factor for the mutual-tough case. According to Lemma 3 , if for all $\delta \in\left(\delta_{\mathrm{TT}}, 1\right)$, there exists a unique symmetric TTIR strategy profile that can be supported as an equilibrium, then the function $p^{*}(\delta)$ decreases monotonically in $\delta \in\left(\delta_{\mathrm{TT}}, 1\right)$. Moreover, it is clear from (12) that $p^{*}(\delta)$ is a continuous function. Combining these features, we conclude that

$$
\lim _{\delta \rightarrow \delta_{\mathrm{TT}}} p^{*}(\delta)=1
$$

since otherwise we could have found a lower discount factor such that $p^{*}(\delta)$ is still less than 1. Equation (20) says that in the limit when $\delta$ tends to the critical discount factor, the punishment of the TTIR strategy becomes most severe as $p^{*}(\delta)$ tends to 1 .

Furthermore, combining (20) and the second equality of (11), we obtain

$$
\lim _{\delta \rightarrow \delta_{\mathrm{TT}}}\left[V^{\mathrm{L}}(\delta)-t-\delta V^{*}(\delta)\right]=0 .
$$

Equation (21) states that the no-deviation condition at the bad turn must be binding at the critical discount factor $\delta_{\mathrm{TT}}$. Using these results, we can determine the critical discount factor as a function of the payoff parameters of the stage game.

Lemma 4 For the repeated game $G_{\infty}$ with $t>l$, if for all $\delta \in\left(\delta_{\mathrm{TT}}, 1\right)$, there exists a unique symmetric TTIR strategy profile that can be supported as a subgame-perfect equilibrium, then the critical discount factor $\delta_{\mathrm{TT}}$ depends on the stage-game parameters according to

$$
\delta_{\mathrm{TT}}=\frac{t-l}{h-t} .
$$

Lemma 4 shows that the critical discount factor for the mutual-tough case depends on the primitives of the stage game in a simple and intuitive way. When the discount factor $\delta$ is sufficiently close to 1 (and future payoffs are important), the no-deviation condition (9) is non-binding. According to Lemma 3, the endogenously determined $p^{*}$ of the TTIR strategy is strictly decreasing in $\delta$ (between $\delta_{\mathrm{TT}}$ and 1). As $\delta$ decreases (and future payoffs become less important), to ensure that the no-deviation condition (9) holds, $p^{*}$ must increase to make deviation more costly. However, the maximum possible punishment is when $p^{*}$ tends to 1 . This defines the critical discount factor $\delta_{\mathrm{TT}}$. As $\delta$ tends to $\delta_{\mathrm{TT}}, p^{*}(\delta)$ tends to 1 according to (20). Moreover, the no-deviation condition at the bad turn becomes binding according to (21), and the punishment approaches the Nash punishment (of choosing $T$ with probability 1 at every period). Thus, as $\delta$ approaches $\delta_{\mathrm{TT}}, V^{*}(\delta)$ approaches $t+\delta_{\mathrm{TT}} t+\delta_{\mathrm{TT}}^{2} t+\ldots=\frac{t}{1-\delta_{\mathrm{TT}}}$. Substituting this result and (7) into (21) lead to

$$
\frac{l+\delta_{\mathrm{TT}} h}{1-\delta_{\mathrm{TT}}^{2}}=\lim _{\delta \rightarrow \delta_{\mathrm{TT}}} V^{\mathrm{L}}(\delta)=\lim _{\delta \rightarrow \delta_{\mathrm{TT}}}\left[t+\delta V^{*}(\delta)\right]=t+\delta_{\mathrm{TT}}\left(\frac{t}{1-\delta_{\mathrm{TT}}}\right) .
$$


Simplifying (21a), we can determine the critical discount factor as in (22).

Having determined $\delta_{\mathrm{TT}}$ according to (22), we now show that for the mutual-tough case, for all $\delta \in\left(\delta_{\mathrm{TT}}, 1\right)$, there exists a unique TTIR strategy profile that can be supported as an equilibrium and it is symmetric.

Proposition 2 For the repeated game $G_{\infty}$ with $t>l$, there exists a unique TTIR strategy profile that can be supported as a subgame-perfect equilibrium if and only if $\delta \in\left(\delta_{\mathrm{TT}}, 1\right)$, where $\delta_{\mathrm{TT}}$ is given by (22). When $\delta \in\left(\delta_{\mathrm{TT}}, 1\right)$, the equilibrium profile is symmetric, with each player's equilibrium randomization probability given by (13) if $a=0$ in (15) or by (14) if $a \neq 0$ in (15).

Proposition 2 shows that for the TTIR strategy to constitute a subgame-perfect equilibrium in the mutual-tough case, the discount factor must be larger than the critical discount factor $\delta_{\mathrm{TT}}=\frac{t-l}{h-t}$. If the players are not sufficiently patient (with the discount factor smaller than $\delta_{\mathrm{TT}}$ ), a player who is supposed to take the bad turn and play $S$ in the turn-taking phase will deviate to play $T$. Thus, the TTIR strategy cannot be supported as an equilibrium when the players are not sufficiently patient. ${ }^{12}$

This is in sharp contrast to the accommodating case, in which the TTIR strategy can be supported as an equilibrium for any discount factor. According to (9), a player who is supposed to take the bad turn according to the TTIR strategy will not deviate if the current gain from deviating, $t-l$, is smaller than the future loss from deviating, $\delta\left(V^{\mathrm{H}}-V^{*}\right)$. When $t<l$ (i.e., the accommodating case), this condition is satisfied for any discount factor because deviation actually yields a current loss, not a gain. When $t>l$ (i.e., the mutual-tough case), however, deviation yields a current gain. If the discount factor is too low, then (9) cannot be satisfied no matter how the randomization probability (which affects a player's intertemporal payoff) is chosen. There is a trade-off between current gain and future loss if a player deviates from the equilibrium strategy. It is this trade-off that distinguishes the analysis and results of the mutual-tough case from those of the accommodating case.

\subsection{Alternative punishment strategies}

In the above analyzes, we assume that any defection during the turn-taking phase will trigger a switch back to the randomization phase, and this randomization phase will continue until randomization gets the players to an asymmetric outcome again. Consider a different TTIR strategy with the following punishment strategy: each player uses the strategy that leads to the symmetric equilibrium of the stage game (i.e., symmetric mixed-strategy equilibrium for the accommodating case, or $(T, T)$ for the mutual-tough case). Another possibility is to punish the player that deviates by restarting the turn-taking path at her bad turn with the other player restarting at her

\footnotetext{
12 Consider a non-standard prisoners' dilemma with $h=6, s=2.3, t=2$ and $l=-1$, which is similar to the game in Dixit and Skeath (1999, p. 359, Fig. 11.2) except for the value of $t$. The stage game parameters satisfy (3) to (5), and $\delta_{\mathrm{TT}}=0.75$ according to (22). If $\delta=0.7$, then it can be shown that a player will deviate when she is supposed to take the bad turn. Thus, the TTIR strategy cannot be supported as an equilibrium when $\delta$ is not high enough.
} 
good turn. This punishment strategy does not treat players symmetrically, but the punishment path is efficient. It can be shown that with either one of these punishment strategies, the no-deviation conditions (8) and (9) during the turn-taking phase are satisfied for all $\delta \in(0,1)$ in the accommodating case, or for $\delta \in\left(\delta_{\mathrm{TT}}, 1\right)$ in the mutual-tough case. More generally, for any credible punishment that deters defection during the turn-taking phase, the analysis in the randomization phase is the same as above. In particular, $p^{*}$ and $V^{*}$, which are related by (11) with symmetric TTIR strategies, are the same as above. Consequently, we obtain the same behavioral implications whenever an alternative punishment strategy prevents players from deviating from equilibrium behavior.

\section{Testable implications}

In this section, we consider two sets of comparative static results, regarding the time in reaching the turn-taking path (for both accommodating and mutual-tough cases) and the critical discount factor (for the mutual-tough case).

\subsection{Delay in reaching the turn-taking path}

When a TTIR profile constitutes an equilibrium, each player chooses $T$ with probability $p^{*}$ in the randomization phase. Therefore, the probability that the players succeed in reaching an asymmetric outcome in a particular period during this phase is given by $2 p^{*}\left(1-p^{*}\right)$. Define the number of time periods taken by the players using the TTIR strategy to reach the turn-taking path as the delay $(D)$. The delay is a geometric random variable and its expected value is

$$
E(D)=\frac{1}{2 p^{*}\left(1-p^{*}\right)} .
$$

We define two concepts related to payoffs $h$ and $l$ in the stage game of $G$. In the stage game, the maximum and minimum amounts of the players' total gain attained as a result of reaching an asymmetric outcome are $h+l-\min \{2 s, 2 t\}$ and $h+l-\max \{2 s, 2 t\}$, respectively. Holding the value of $s$ and $t$ constant, a rise in $h+l$ increases both the maximum and the minimum gains that the players attain when they reach an asymmetric outcome. Therefore, we define

$$
\lambda=h+l
$$

as the efficiency gain parameter when achieving either one of the two asymmetric outcomes. We also define

$$
\theta=\frac{h}{l}
$$

as the conflict of interest parameter. Without loss of generality, we can normalize the payoffs so that $l>0$. As a result, $\lambda>0$ and $\theta>1$ according to (3), (24), and (25). 
Table 3 Two games of chicken which only differ in the value of $\theta$

(a) A game of chicken with $s=90, t=10, \lambda=200$, and $\theta=4$

\begin{tabular}{lll}
\hline $1 \backslash 2$ & Tough & Soft \\
\hline Tough & $(10,10)$ & $(160,40)$ \\
Soft & $(40,160)$ & $(90,90)$
\end{tabular}

(b) A game of chicken with $s=90, t=10, \lambda=200$, and $\theta=9$

\begin{tabular}{lll}
\hline $1 \backslash 2$ & Tough & Soft \\
\hline Tough & $(10,10)$ & $(180,20)$ \\
Soft & $(20,180)$ & $(90,90)$ \\
\hline
\end{tabular}

From (24) and (25), we can obtain $h=\frac{\theta \lambda}{1+\theta}$ and $=\frac{\lambda}{1+\theta}$. While the original specification of the stage game uses the four parameters $s, t, h$, and $l$ as primitives, the game can also be expressed in terms of parameters $s, t, \lambda$, and $\theta$. This is illustrated in the bottom panel of Table 1. This re-specification is useful for some comparative static analysis, as will be shown in Proposition 3 . When the efficiency parameter $\lambda$, as well as $s$ and $t$, are held constant, an increase in $\theta$ implies that there is a higher degree of conflict in the stage game. This can be illustrated in Table 3 involving two games of chicken. The top panel corresponds to the case when $s=90, t=10, \lambda=200$, and $\theta=4$. The game in the bottom panel is obtained from increasing the degree of conflict to $\theta=9$, with the values of the other three parameters held constant.

It is natural to conjecture that an increase in the degree of distributional conflict will induce the players to behave more aggressively in choosing Tough with a higher probability at the randomization phase. As a result, the expected value of delay will be increased. It turns out that this conjecture is correct for most but not all games. The following Proposition shows that an increase in the degree of conflict $(\theta)$ will increase expected delay in the mutual-tough case, but may decrease equilibrium delay in a range of $\theta$ for a number of games (including game of chicken) in the accommodating case.

Proposition 3 (a) For the TTIR strategy profile that constitutes an equilibrium in the repeated game $G_{\infty}$ with $t>l$ and $\delta \in\left(\delta_{\mathrm{TT}}, 1\right)$, the expected delay is always increasing in the degree of conflict $(\theta)$ of the stage game.

(b) For the TTIR strategy profile that constitutes an equilibrium in the repeated game $G_{\infty}$ with $t<l$ and $\delta \in(0,1)$, the expected delay is always increasing in $\theta$ if $t \geq s$. However, if $s>t$, there exist games in which the expected delay is not always increasing in $\theta$.

The intuition of Proposition 3 is as follows. Since a change in the degree of conflict only affects expected delay through its effect on the equilibrium randomization probability, we have $\frac{\partial E(D)}{\partial \theta}=\frac{\partial E(D)}{\partial p^{*}} \times \frac{\partial p^{*}}{\partial \theta}$. We know from (23) that $E(D)$ reaches its minimum at $p^{*}=0.5$. Furthermore, $E(D)$ is decreasing in $p^{*}$ when $0<p^{*}<0.5$, but is increasing in $p^{*}$ when $0.5<p^{*}<1$. Thus, $\frac{\partial E(D)}{\partial p^{*}}>0$ if and only if $0.5<p^{*}<1$. On the other hand, it can be shown that 


$$
\frac{\partial p^{*}}{\partial \theta}=\frac{\frac{\partial V^{\mathrm{H}}}{\partial \theta}-p^{*}\left[\frac{\partial\left(V^{\mathrm{H}}+V^{\mathrm{L}}\right)}{\partial \theta}\right]}{\left(V^{\mathrm{H}}-s-\delta V^{*}\right)+\left(V^{\mathrm{L}}-t-\delta V^{*}\right)+\delta\left(1-2 p^{*}\right) \frac{\partial V^{*}}{\partial p^{*}}},
$$

and the numerator on the right-hand side of (26) is positive. For the denominator, two of the three terms are positive because the no-deviation conditions hold at the equilibrium. The third term, $\delta\left(1-2 p^{*}\right) \frac{\partial V^{*}}{\partial p^{*}}$, is also positive if $0.5<p^{*}<1$. Thus, $\frac{\partial p^{*}}{\partial \theta}>0$ if $0.5<p^{*}<1$. Combining these results, we conclude that $0.5<p^{*}<1$ is a sufficient condition for $\frac{\partial E(D)}{\partial \theta}>0$.

For the mutual-tough case and the accommodating case with $t \geq s$, it can be shown that $0.5<p^{*}<1$. In these games, the payoff parameters are such that each player chooses Tough with an equilibrium probability higher than 0.5 in the randomization phase to ensure that her opponent is indifferent in choosing Tough or Soft. When $\theta$ increases, the intertemporal efficiency gain from turn taking is more unevenly distributed, and it becomes more attractive to be the first player to take the good turn. Thus, each player behaves more aggressively in choosing Tough with a higher probability. Since the increase in equilibrium randomization probability occurring in the range $(0.5,1)$ means that each player's probability of playing Tough is further away from 0.5 , the probability of reaching the turn-taking path in a particular period, given by $2 p^{*}\left(1-p^{*}\right)$, is reduced. As a result, expected delay increases because of the players' more aggressive behavior.

The condition $0.5<p^{*}<1$ is not always satisfied in the accommodating case when $s>t$. We have performed computational analysis for these games and found counter-intuitive pattern that $E(D)$ is not always increasing in $\theta$. We present one such counter-example as an illustration. Consider the games of chicken with $s=90, t=10$, $\lambda=200, \delta=0.6$ and $\theta$ ranging from 2 to 10 . In all these games, $h>s>l>t$. Figure 1 shows that when $\theta$ is relatively small, the equilibrium randomization probability is less than 0.5. This is because the payoff at the outcome (Tough, Tough) is relatively unattractive in these games of chicken, and each player chooses Tough with a probability less than 0.5 to ensure that her opponent is willing to randomize between $T$ and $S$. In this example, when $\theta$ increases and being the first player to take the good turn becomes more attractive, the equilibrium value $p^{*}$ increases. However, since $0<p^{*}<0.5$ when $\theta$ is relatively small, an increase in $p^{*}$ means that it is getting closer to 0.5 and thus, expected delay will decrease. This explains the counterintuitive result. On the other hand, when $\theta$ is relatively large and the efficiency gain of turn taking is more unevenly distributed, even though a player's current-period payoff at the outcome (Tough, Tough) is still unattractive, the higher level of $\theta$ will tilt the trade-off of choosing $T$ versus $S$ in favor of the former. As a result, $0.5<p^{*}<1$. In this range of $\theta$, the positive relationship between $E(D)$ and $\theta$ is qualitatively the same as the mutual-tough case. Combining the above results, we observe the U-shaped pattern between $E(D)$ and $\theta$ for the games of chicken in Figure 2. ${ }^{13}$

13 Note that the variation of $E(D)$ with respect to $\theta$ in Fig. 2 is quite small. This is because we choose the parameters of the games of chicken to illustrate the non-monotonic relationship of $E(D)$ with respect to $\theta$, and thus $p^{*}$ moves from below 0.5 to above 0.5 . Since the values of $p^{*}$ in Fig. 2 are close to 0.5 , 


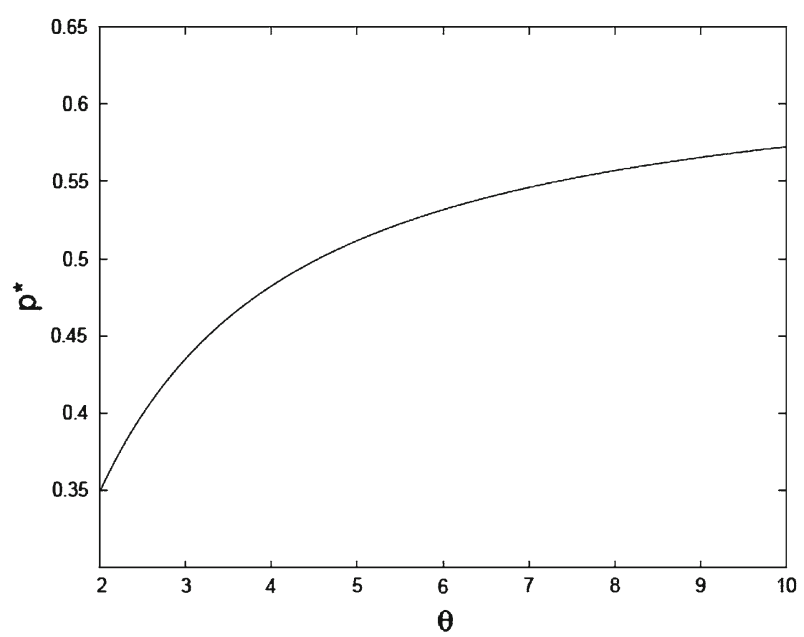

Fig. $1 p^{*}$ as a function of $\theta$ (for games of chicken)

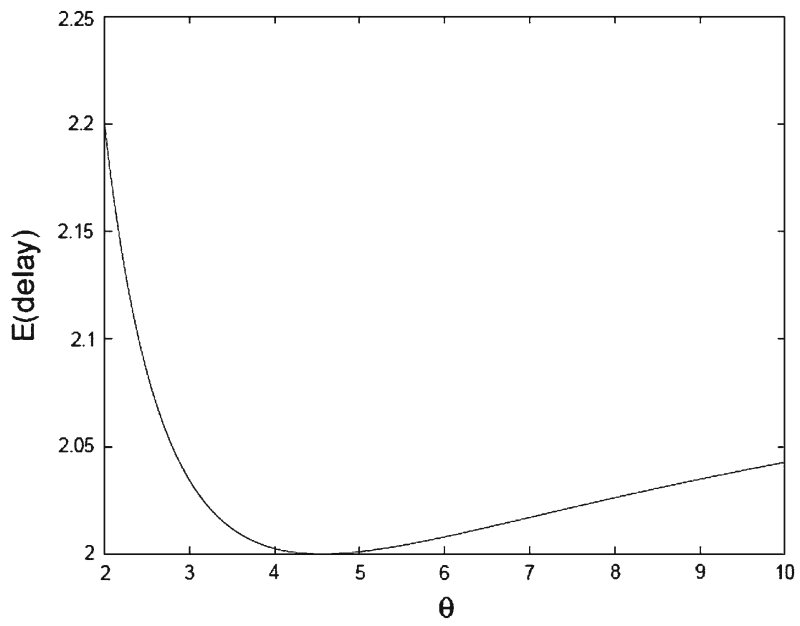

Fig. 2 Expected value of delay as a function of $\theta$ (for games of chicken)

\subsection{Critical discount factor (for the mutual-tough case)}

The critical discount factor $\delta_{\text {TT }}$ for the mutual-tough case is given in Lemma 4 . We first examine how the critical discount factor is affected by a change in parameter $t$. Note that the left-hand term of (21a) is a player's intertemporal payoff of adhering to the equilibrium strategy when her bad turn comes up, whereas the right-hand term is the intertemporal payoff of defecting. An increase in $t$ (at an unchanged $\delta_{\mathrm{TT}}$ ) will increase the current and future payoffs of defecting. To restore the equilibrium condition (21a),

the corresponding values of $E(D)$ are close to 2 and do not vary much. We have also found other games in which the changes in expected delay with respect to $\theta$ are more substantial and are monotonic. Those games would be more useful for designing experiments to test the theoretical predictions in this article. 
the critical discount factor has to increase. Formally, differentiating $\delta_{\text {TT }}$ with respect to $t$ gives

$$
\frac{\partial \delta_{\mathrm{TT}}}{\partial t}=\frac{h-l}{(h-t)^{2}}>0,
$$

because of (3) and (5). Holding the value of $h$ and $l$ constant, an increase in $t$ (up to $\frac{h+l}{2}$ ) causes $\delta_{\mathrm{TT}}$ to rise. As a result, the interval $\left(\delta_{\mathrm{TT}}, 1\right)$ becomes narrower, and it is less likely that the TTIR strategy can be supported as an equilibrium.

We also study how changes in the degree of conflict and the efficiency gain affect the critical discount factor for the mutual-tough case. The critical discount factor in (22) can be expressed as a function of $t, \theta$, and $\lambda$ as follows:

$$
\delta_{\mathrm{TT}}=\frac{t-l}{h-t}=\frac{(1+\theta) t-\lambda}{\theta \lambda-(1+\theta) t} .
$$

Because $t>l>0$ and $\lambda=h+l>2 t$, we have

$$
\frac{\partial \delta_{\mathrm{TT}}}{\partial \theta}=\frac{\lambda(\lambda-2 t)}{[\theta \lambda-(1+\theta) t]^{2}}>0,
$$

and

$$
\frac{\partial \delta_{\mathrm{TT}}}{\partial \lambda}=\frac{-t\left(\theta^{2}-1\right)}{[\theta \lambda-(1+\theta) t]^{2}}<0 .
$$

Equation (28) says that $\delta_{\mathrm{TT}}$ is increasing in $\theta$, which means that an increase in the degree of conflict makes it less likely that the TTIR strategy can be supported as an equilibrium. An increase in distributional conflict (when other parameters are unchanged) implies that the player's payoff at the bad turn during the turn-taking phase is relatively unattractive. As a result, the players have a greater incentive to defect (at a given discount factor), and supporting the TTIR strategy as an equilibrium requires a higher critical discount factor.

Equation (29) says that $\delta_{\mathrm{TT}}$ is decreasing in $\lambda$, which means that a decrease in the efficiency gain from achieving either of the asymmetric outcomes (provided that (4) and (5) still hold) leads to an increase in $\delta_{\mathrm{TT}}$, and makes it less likely that the TTIR strategy can be supported as an equilibrium.

We summarize the above results in the following Proposition.

Proposition 4 For the repeated game $G_{\infty}$ with $t>l$ and $\delta \in\left(\delta_{\mathrm{TT}}, 1\right)$, there exists a unique TTIR strategy profile that can be supported as an equilibrium. An increase in $t$, an increase in $\theta$, or a decrease in $\lambda$ will lead to a higher critical discount factor $\delta_{\mathrm{TT}}$, and make it less likely that the TTIR strategy can be supported as an equilibrium.

\section{Concluding remarks}

Motivated by the importance of turn-taking behavior in many field and experimental settings, this article develops an analysis of turn taking in a repeated symmetric $2 \times 2$ 
game that nests many widely studied games as special cases. Our specification of one-period alternation in the TTIR strategies, and our analysis on the delay in reaching the turn-taking path, are inspired by experimental findings. The unified analysis of turn-taking behavior in these well-known games generates novel testable implications, which provides guidance for new laboratory experiments examining how differences in the structure of the strategic environment affect the effectiveness of turn taking as a mechanism for intertemporal cooperation. For example, our analysis predicts that the expected delay in reaching the turn-taking path is always increasing in the degree of conflict of the stage game for the mutual-tough case. This prediction and related ones for the accommodating case can be tested in controlled laboratory experiments that systematically vary the degree of conflict.

In this article, we analyze turn taking in a benchmark model that does not allow for communication. However, the results reported in Ostrom et al. (1994) suggest that nonbinding communication can be efficiency-enhancing in the laboratory repeated games. On the other hand, the experimental results in Prisbrey (1992) suggest that asymmetric turn-taking schemes-for example, one in which a player is supposed to take the good turn for two periods and then take the bad turn for one period, with her opponent doing the opposite - are more difficult to sustain. In the future, we plan to investigate whether extending the model to incorporate different kinds of asymmetry and/or communication will make it easier or more difficult for the (potentially more sophisticated) turn-taking strategies to achieve intertemporal cooperation.

Finally, the model considered in this article rules out the possibility that a player may attempt to "modify the game" to her advantage. However, in environments related to deciding whether turn taking can be used to determine, for example, who will be the chairperson of a department or which nation will chair an important international committee, players may be reluctant to take the bad turn because they are concerned that the player who gets to take the good turn in a particular period may attempt to alter the game to her advantage. A fruitful direction for future research is to investigate, in such an environment, when and how some kind of turn-taking strategies may still be able to mitigate the coordination and conflict problems that are more difficult than the one considered in our benchmark model.

Acknowledgments We are grateful to Stephen Chiu, Simon Grant, Xianming Zhou, two anonymous referees, and seminar participants at the Australian National University, City University of Hong Kong, McGill University, Monash University, Purdue University, and University of Hong Kong for helpful comments, and to Daniel Kelly and Philip Ng for excellent research assistance. We thank the Research Grants Council of Hong Kong (project HKU7223/04H) for financial support.

Open Access This article is distributed under the terms of the Creative Commons Attribution Noncommercial License which permits any noncommercial use, distribution, and reproduction in any medium, provided the original author(s) and source are credited.

\section{Appendix}

Proof of Proposition 1 We are going to prove that, first, the solution to the two conditions in (12) exists and, second, it is unique and symmetric. We then show that this solution satisfies (8) and (9). 
Existence. It suffices to prove the existence of symmetric TTIR strategies with $p_{1}=p_{2}=p$ that satisfy (12). To apply well-known mathematical results, we extend the domain of $p$ from $(0,1)$ to $[0,1]$. We define the continuous function

$$
f(p)=\frac{V^{\mathrm{H}}-s-\delta V(p)}{\left[V^{\mathrm{H}}-s-\delta V(p)\right]+\left[V^{\mathrm{L}}-t-\delta V(p)\right]}
$$

over $p \in[0,1]$, where $V(p)=V_{i}(p, p)$ is defined according to (10). It is easy to observe that the symmetric solution to (12) is a fixed point of the function $f$ (.) in (A1).

According to Lemma 1 and Lemma 2, when $t<l$, (8a) and (9a) hold for any $p \in(0,1)$. We conclude from (A1) that $0<f(p)<1$. Moreover, it is easy to show that $0<f(0)<1$ and $0<f(1)<1$. Therefore, $f($.) is a continuous function from the compact set $[0,1]$ to itself. Applying the Brouwer's Fixed Point Theorem, we conclude that this function has a fixed point. That is, there exists a $p \in[0,1]$ such that $f(p)=p$. Moreover, since $f(0)>0$ and $f(1)<1$, we conclude that $f(p)=p$ does not hold at $p=0$ or $p=1$, and the solution to (12) exists in the open interval $(0,1)$.

Uniqueness and symmetry. We now establish that only one pair of $\left(p_{1}^{*}, p_{2}^{*}\right)$ satisfies the conditions in (12), and that the unique equilibrium is symmetric $\left(p_{1}^{*}=p_{2}^{*}\right)$. Note that (11) leads to

$$
V_{i}^{*}=\frac{p_{j}^{*} t+\left(1-p_{j}^{*}\right) V^{\mathrm{H}}}{1-\delta p_{j}^{*}}=\frac{p_{j}^{*} V^{\mathrm{L}}+\left(1-p_{j}^{*}\right) s}{1-\delta\left(1-p_{j}^{*}\right)}
$$

Substituting (6) and (7) into the second equality of (A2) and simplifying give

$$
a\left(p_{j}^{*}\right)^{2}+b p_{j}^{*}+c=0,
$$

where $j=1,2$ and $a, b$ and $c$ are given in (15) to (17). ${ }^{14}$ From (15) to (17), we know that $c>0$ but that $a$ and $b$ can be either positive, negative or zero.

Since (A3) is a quadratic equation in $p_{j}^{*}$, there are at most two real roots. Together with the existence result above, there must be either one or two $p_{j}^{*}$ in the interval $(0,1)$. From standard results for quadratic equations, we know that if $a=0$, then there is just one $p_{j}^{*}$ and it is given by (13). If $a \neq 0$, the two roots to (A3) are given by (14) and

$$
p_{j}^{*}=\frac{-b+\sqrt{b^{2}-4 a c}}{2 a} .
$$

\footnotetext{
14 A common approach to show the uniqueness of equilibrium is to use the Contraction Mapping Theorem. We have, however, found some counterexamples for the mutual-tough case (such as $h=160, l=40, t=$ $80, s=20$ and $\delta=0.75$ ) that $f$ in (A1) is not a contraction mapping. Hence, we use a different approach.
} 
Since at least one $p_{j}^{*}$ lies in $(0,1)$, either $(14)$ or (A4), but not both, may lie outside the interval $(0,1)$.

We can further show that (a) if $a<0$, then $p_{j}^{*}$ in (A4) is negative; (b) if $a>0$ and $b>0$, then $p_{j}^{*}$ in (A4) is negative; (c) if $a>0$ and $b<0$, then $p_{j}^{*}$ in (A4) is larger than 1 for all $\delta \in(0,1)$; and (d) $b=0$ is inconsistent with $a>0$ and $b^{2}-4 a c \geq 0$. Therefore, whether $a$ in (15) is positive or negative, $p_{j}^{*}$ in (A4) does not lie in the interval $(0,1)$.

Consequently, there is only one solution to (12) that satisfies $0<p_{j}^{*}<1$, and the solution is given by (13) if $a=0$ or (14) if $a \neq 0$. Moreover, $p_{1}^{*}=p_{2}^{*}=p^{*}$ since both of them are defined according to (13) or (14).

Finally, applying Lemma 1 and Lemma 2, it is easy to see that the unique solution to (12) also satisfies the no-deviation conditions (8) and (9). We conclude that there is a unique TTIR strategy profile that can be supported as an equilibrium for $G_{\infty}$ with $t<l$ for all $\delta \in(0,1)$. Moreover, it is symmetric.

\section{References}

Athey, S., \& Bagwell, K. (2008). Collusion with persistent cost shocks. Econometrica, 76, 493-540.

Bergerud, E. M. (1993). Red thunder, tropic lightning: The world of a combat division in Vietnam. Boulder: Westview Press Inc.

Berkes, F. (1992). Success and failure in marine coastal fisheries of Turkey. In D. W. Bromley (Ed.), Making the commons work: Theory, practice, and policy (pp. 161-182). San Francisco: Institute for Contemporary Studies.

Bhaskar, V. (2000). Egalitarianism and efficiency in repeated symmetric games. Games and Economic Behavior, 32, 247-262.

Bornstein, G., Budescu, D., \& Zamir, S. (1997). Cooperation in intergroup, N-person, and two-person games of chicken. Journal of Conflict Resolution, 41, 384-406.

Dixit, A., \& Skeath, S. (1999). Games of strategy. New York: W. W. Norton \& Company.

Friedman, J. W. (1994). Introduction and overview. In J. W. Friedman (Ed.), Problems of coordination in economic activity (pp. 3-15). Boston: Kluwer Academic Publishers.

Harrison, G., \& Hirshleifer, J. (1989). An experimental evaluation of weakest link/best shot models of public goods. Journal of Political Economy, 97, 201-225.

Helbing, D., Schönhof, M., Stark, H.-U., \& Holyst, J. A. (2005). How individuals learn to take turns: Emergence of alternating cooperation in a congestion game and the prisoner's dilemma. Advances in Complex Systems, 8, 87-116.

Kaplan, T. R., \& Ruffle, B. J. (2007). Which way to cooperate? Working paper. University of Exeter and Ben-Gurion University

Lau, S.-H. P., \& Mui, V.-L. (2008). Using turn taking to mitigate coordination and conflict problems in the repeated battle of the sexes game. Theory and Decision, 65, 153-183.

Luce, R. D., \& Raiffa, H. (1957). Games and decisions: Introduction and critical survey. New York: Wiley.

Ostrom, E. (1990). Governing the commons: The evolution of institutions for collective action. New York: Cambridge University Press.

Ostrom, E., Gardner, R., \& Walker, J. (with A. Agrawal, W. Blomquist, E. Schlager, and S. Y. Tang). (1994). Rules, games, and common-pool resources. Ann Arbor: University of Michigan Press.

Prisbrey, J. (1992). Cooperation in reciprocity games and in the voluntary contributions mechanism. Ph.D. Dissertation, California Institute of Technology.

Skrzypacz, A., \& Hopenhayn, H. (2004). Tacit collusion in repeated auctions. Journal of Economic Theory, 114, 153-169.

Ward, H. (1998). A game theoretic analysis of the politics of taking it in turns. British Journal of Political Science, 28, 355-387. 